\title{
Analytical Solutions of System of Non-Linear Differential Equations in the Single-Enzyme, Single-Substrate Reaction with Non-Mechanism-Based Enzyme Inactivation
}

\author{
Govindhan Varadharajan, Lakshmanan Rajendran* \\ Department of Mathematics, The Madura College, Madurai, India \\ E-mail:"raj_sms@rediffmail.com \\ Received June 23, 2011; revised July 15, 2011; accepted July 23, 2011
}

\begin{abstract}
A closed form of an analytical expression of concentration in the single-enzyme, single-substrate system for the full range of enzyme activities has been derived. The time dependent analytical solution for substrate, enzyme-substrate complex and product concentrations are presented by solving system of non-linear differential equation. We employ He's Homotopy perturbation method to solve the coupled non-linear differential equations containing a non-linear term related to basic enzymatic reaction. The time dependent simple analytical expressions for substrate, enzyme-substrate and free enzyme concentrations have been derived in terms of dimensionless reaction diffusion parameters $\varepsilon, \lambda_{1}, \lambda_{2}$ and $\lambda_{3}$ using perturbation method. The numerical solution of the problem is also reported using SCILAB software program. The analytical results are compared with our numerical results. An excellent agreement with simulation data is noted. The obtained results are valid for the whole solution domain.
\end{abstract}

Keywords: Non-Linear Reaction Equations, Enzyme Inactivation, Homotopy Perturbation Method, Time Dependent Analytical Solution

\section{Introduction}

An enzyme is a biological catalyst that regulates the rate of chemical reaction in a living organism. Enzymes are very essential as most chemical reactions would occur too slowly or would lead to different product without the activity of enzymes. Enzymes bond with a substrate to form a transient state, an unstable intermediate complex that requires less energy for the reaction to proceed. Like any catalyst, the enzyme remains unaltered by the completed reaction and can therefore continue to interact with substrates. Enzymes may speed up reactions by a factor of many millions. Under temperature changes [1], diluted conditions, or changes in the reaction medium ( $\mathrm{pH}$ or buffer) [2,3], enzyme can undergo progressive loss of activity. Actually enzyme inactivation can result in grievous errors in describing the behaviour of the system, such as incorrect estimation of the kinetic parameters. Therefore it is very important to be able to know when enzyme inactivation is affecting a reaction. Under- standing the effects of enzyme inactivation is important for application such as predicting the behaviour of chemical reactions in the food, chemical, and pharmaceutical industries $[4,5]$. While there are numerous models available for mechanism-based inactivation systems or suicide substrates [6,7], there is currently no methodology that yields quantitative predictions for non-mechanism-based enzyme inactivation. In this paper we derive an expression for concentration of substrate, enzymesubstrate complex and product with non-mechanismbased enzyme inactivation, interms of dimensionless reaction diffusion parameters $\varepsilon, \lambda_{1}, \lambda_{2}$ and $\lambda_{3}$ using Homotopy perturbation method (HPM) and comparative study of the same with numerical simulation.

\section{Mathematical Formulation of the Problem}

The model of biochemical reaction was set forth by Michaelis and Menten in 1913 [8] and further developed by Briggs and Haldane in 1925 [9]. This formulation 
considers a reaction where a substrate $S$ binds an enzyme $E$ reversibly to form a complex $C$. The complex can then decay irreversibly to a product $P$ and the enzyme, which is then free to bind another molecule of the substrate. The enzyme is normally considered more stable while incorporated in $C$ than when in free $E$ form. From this, we can add to the Michaelis-Menten mechanism that free $E$ decays into its inactive form $E_{i}$. This single enzyme-substrate reaction system is represented as follows [10]:

$$
\begin{gathered}
S+E \underset{k_{-1}}{\stackrel{k_{1}}{\Leftrightarrow}} C \stackrel{k_{\text {cat }}}{\rightarrow} P+E \\
E \stackrel{k_{3}}{\rightarrow} E_{i}
\end{gathered}
$$

In this mechanism, the enzyme inactivation is irreversible. This mechanism illustrates the binding of substrate $S$ and release of product $P$. $E$ is the free enzyme and $C$ is the enzyme-substrate complex. The time evolution of reaction (1)-(2) are obtained by applying the law of mass action to yield the set of system of following non-linear differential equations.

$$
\begin{gathered}
\frac{\mathrm{d} s}{\mathrm{~d} t}=k_{1}\left(-e s+K_{S} c\right) \\
\frac{\mathrm{d} e}{\mathrm{~d} t}=k_{1}\left(-e s+K_{M} c-K_{\delta} e\right) \\
\frac{\mathrm{d} c}{\mathrm{~d} t}=k_{1}\left(e s-K_{M} c\right) \\
\frac{\mathrm{d} p}{\mathrm{~d} t}=k_{c a t} c \\
\frac{\mathrm{d} e_{i}}{\mathrm{~d} t}=k_{1} K_{\delta} e
\end{gathered}
$$

Initial conditions at $t=0$ is given by

$$
s=s_{0}, e=e_{0}, c=0, p=0, e_{i}=0 .
$$

In this system, $K_{S}$ is the equilibrium dissociation constant for enzyme-substrate complex, $K_{M}$ is the Michaelis-Menten constant and $K_{\delta}$ is the constant for enzyme inactivation. By imposing the following two conservation of laws,

$$
e_{0}=e+c+e_{i}
$$

and

$$
s_{0}=s+c+p
$$

We describe the reaction mechanism (3)-(7) using the following differential equations

$$
\begin{aligned}
& \frac{\mathrm{d} s}{\mathrm{~d} t}=-k_{1} s\left(e_{0}-c-e_{i}\right)+k_{1} K_{S} c \\
& \frac{\mathrm{d} c}{\mathrm{~d} t}=k_{1} s\left(e_{0}-c-e_{i}\right)-k_{1} K_{M} c \\
& \frac{\mathrm{d} e_{i}}{\mathrm{~d} t}=k_{1} K_{\delta}\left(e_{0}-c-e_{\mathrm{i}}\right)
\end{aligned}
$$

with initial conditions at

$$
t=0, s=s_{0}, c=0, e_{i}=0
$$

By introducing the following set of dimensionless parameters

$$
\begin{aligned}
& \tau=\frac{k_{1} e_{0} t}{\varepsilon}, u(\tau)=\frac{\mathrm{s}(t)}{s_{0}}, \mathrm{v}(\tau)=\frac{c(t)}{e_{0}}, \\
& w(\tau)=\frac{e_{\mathrm{i}}(t)}{e_{0}}, \lambda_{1}=\frac{K_{S}}{s_{0}}, \lambda_{2}=\frac{K_{M} \varepsilon}{e_{0}}, \\
& \lambda_{3}=\frac{K_{\delta} \varepsilon}{e_{0}}, \varepsilon=\frac{e_{0}}{s_{0}}
\end{aligned}
$$

The system of Equations (11)-(13) with initial condition (14) can be represented in dimensionless form as follows:

$$
\begin{gathered}
\frac{\mathrm{d} u}{\mathrm{~d} \tau}=-u \varepsilon+\varepsilon u v+\varepsilon u w+\lambda_{1} \varepsilon v \\
\frac{\mathrm{d} v}{\mathrm{~d} \tau}=u-u v-u w-\lambda_{2} v \\
\frac{\mathrm{d} w}{\mathrm{~d} \tau}=\lambda_{3}-\lambda_{3} v-\lambda_{3} w
\end{gathered}
$$

The boundary conditions are

$$
u(0)=1, v(0)=0, w(0)=0
$$

\section{Analytical Expressions of Concentrations under Non-Steady State and Steady State Condition}

Nonlinear phenomena play a crucial role in applied mathematics and physics. Explicit solutions to the nonlinear equations are of fundamental importance. Various methods for obtaining explicit solution to nonlinear evolution equations have been proposed. Recently, many authors have used the HPM for solving various problems and demonstrated the efficiency of the HPM for solving non-linear structures and various physics and engineering problems [11-14]. This method is a combination of topology and classic perturbation techniques. Ji Huan He used the HPM to solve the Lighthill equation [15], the Duffing equation [16] and the Blasius equation [17]. The idea has been used to solve non-linear boundary value problems, integral equations and many other problems. In these methods [18-22], the homotopy perturbation method is applied and the obtained results show that the HPM is very effective and simple. The HPM is unique in its applicability, accuracy, efficiency and uses the imbedding parameter $p$ as a small parameter and only a few iterations are needed to search for an asymptotic solution. Using this method, we can obtain the following solution to Equations (15)-(17) (Ref Appendix-A) 


$$
\begin{gathered}
u(\tau)=e^{-\varepsilon \tau}+\frac{\lambda_{1} \varepsilon \tau e^{-\varepsilon \tau}}{\lambda_{2}-\varepsilon}+\frac{\lambda_{1} \varepsilon\left[e^{-\lambda_{2} \tau}-e^{-\varepsilon \tau}\right]}{\left(\lambda_{2}-\varepsilon\right)^{2}}+\varepsilon \tau e^{-\varepsilon \tau}+\frac{\varepsilon\left[e^{-\left(\lambda_{3}+\varepsilon\right) \tau}-e^{-\varepsilon \tau}\right]}{\lambda_{3}} \\
v(\tau)=\frac{e^{-\lambda_{2} \tau}}{\varepsilon\left(\lambda_{2}-\varepsilon\right)}-\frac{e^{-\left(\lambda_{2}+\varepsilon\right) \tau}}{\varepsilon\left(\lambda_{2}-\varepsilon\right)}+\frac{e^{-\lambda_{2} \tau}}{\left(\lambda_{2}-\varepsilon\right)\left(\lambda_{2}-2 \varepsilon\right)}-\frac{e^{-2 \varepsilon \tau}}{\left(\lambda_{2}-\varepsilon\right)\left(\lambda_{2}-2 \varepsilon\right)}+\frac{\left[e^{-\left(\lambda_{3}+\varepsilon\right) \tau}-e^{-\lambda_{2} \tau}\right]}{\lambda_{2}-\lambda_{3}-\varepsilon} \\
w(\tau)=1-e^{-\lambda_{3} \tau}+\frac{\lambda_{3} e^{-\lambda_{3} \tau}}{\left(\lambda_{2}-\varepsilon\right)\left(\lambda_{3}-\varepsilon\right)}-\frac{\lambda_{3} e^{-\varepsilon \tau}}{\left(\lambda_{2}-\varepsilon\right)\left(\lambda_{3}-\varepsilon\right)}+\frac{\lambda_{3} e^{-\lambda_{2} \tau}}{\left(\lambda_{2}-\varepsilon\right)\left(\lambda_{3}-\lambda_{2}\right)}-\frac{e^{-\lambda_{3} \tau}}{\left(\lambda_{2}-\varepsilon\right)\left(\lambda_{3}-\lambda_{2}\right)}
\end{gathered}
$$

Equations (19)-(21) represent the analytical expression of the dimensionless substrate concentration $u(\tau)$, enzyme-substrate concentration $v(\tau)$ and free enzyme concentration $w(\tau)$ for all values of parameters $\varepsilon, \lambda_{1}, \lambda_{2}$ and $\lambda_{3}$.

For steady state condition, the differential Equations (15)-(17) become as follows:

$$
\begin{gathered}
-u \varepsilon+\varepsilon u v+\varepsilon u w+\lambda_{1} \varepsilon v=0 \\
u-u v-u w-\lambda_{2} v=0 \\
\lambda_{3}-\lambda_{3} v-\lambda_{3} w=0
\end{gathered}
$$

Solving the above equations, we can obtain substrate concentration $u$, enzyme substrate concentration $v$ and free enzyme concentration $w$, as follows:

$$
u=0, v=0, w=1
$$

When $t$ tends to infinity, the analytical expression corresponding to the substrate concentration $u$, enzyme substrate concentration $v$ and free enzyme concentration $w$ from the Equations (19)-(21) confirms the validity of mathematical analysis.

\section{Results and Discussion}

The non-linear differential Equations (15)-(18), are also solved using numerical methods. The functionbvp $4 \mathrm{c}$ in Scilab software which is a function of solving two-point boundary value problems (BVPs) for ordinary differential equations is used to solve this equation. Its numerical solution is compared with our analytical result in Figures 1-5 and it gives a satisfactory agreement for all values of time $\tau$. The Scilab program is also given in Appendix B.

Equations (19)-(21) are the closed and simple analytical expression of concentrations. Figures 1-5 describes the dimensionless unsteady-state concentrations versus time $\tau$ The analytical expression of concentrations of substrate $u(\tau)$, enzyme-substrate complex $v(\tau)$ and free enzyme $w(\tau)$ have been plotted for various values of dimensionless reaction parameters $\varepsilon, \lambda_{1}, \lambda_{2}$ and $\lambda_{3}$. Recently Schnell and Hanson [10] obtained the following analytical expression of concentration of enzyme substrate complex $c$ and free enzyme $e_{i}$ assuming

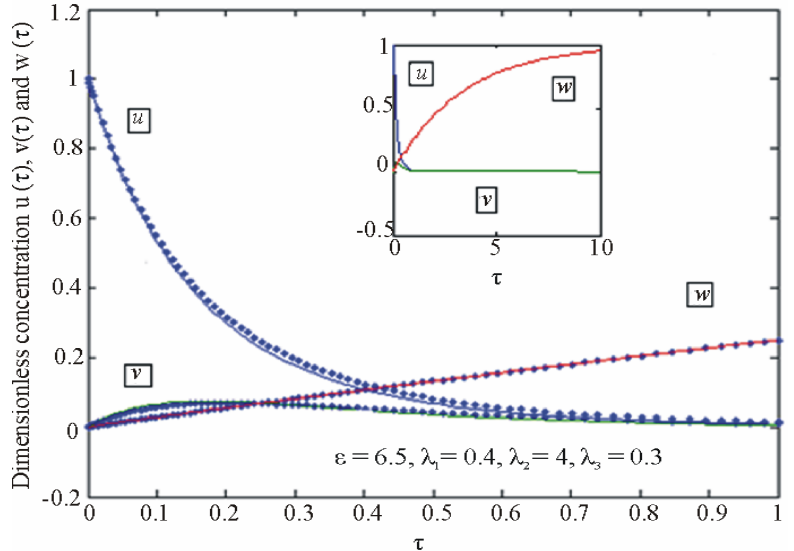

Figure 1. Time-dependent behaviour of the reactant concentration of the substrate $u(\tau)$, enzyme-substrate $v(\tau)$ and free enzyme $w(\tau)$ for $\varepsilon=6.5, \lambda_{1}=0.4, \lambda_{2}=4$ and $\lambda_{3}=0.3$. The curves are plotted using Equations (19)-(21). The key to the graph (---) represents Equations (19)-(21) and (....) denotes simulation.

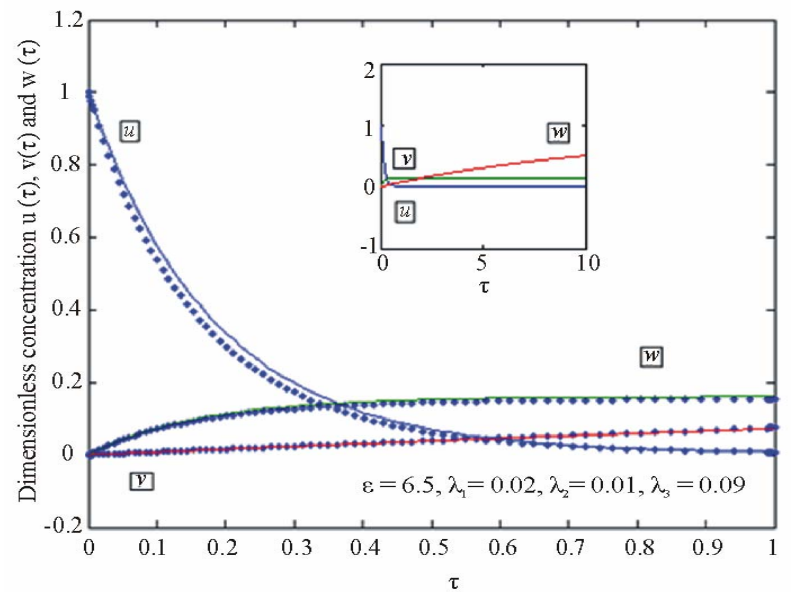

Figure 2. Time-dependent behaviour of the reactant concentration of the substrate $\boldsymbol{u}(\tau)$, enzyme-substrate $v(\tau)$ and free enzyme $w(\tau)$ for $\varepsilon=6.5, \lambda_{1}=0.02$, $\lambda_{2}=0.01$ and $\lambda_{3}=0.09$.The curves are plotted using Equations (19)-(21). The key to the grap (----) represents Equations (19)-(21) and (....) denotes simulation. 


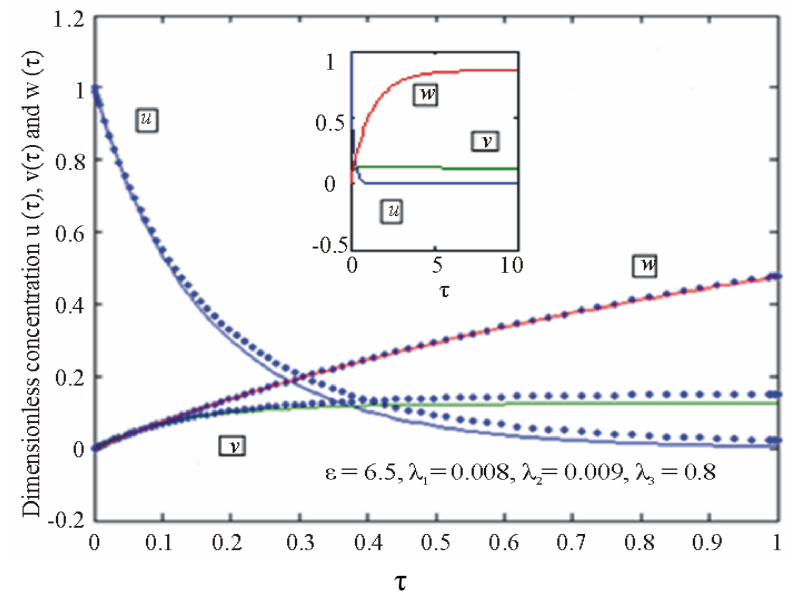

Figure 3. Time-dependent behaviour of the reactant concentration of the substrate $u(\tau)$, enzyme-substrate $v(\tau)$ and free enzyme $w(\tau)$ for $\varepsilon=6.5, \lambda_{1}=0.008$,

$\lambda_{2}=0.009$ and $\lambda_{3}=0.8$. The curves are plotted using Equations (19)-(21). The key to the graph (---) represents Equations (19)-(21) and (....) denotes simulation.

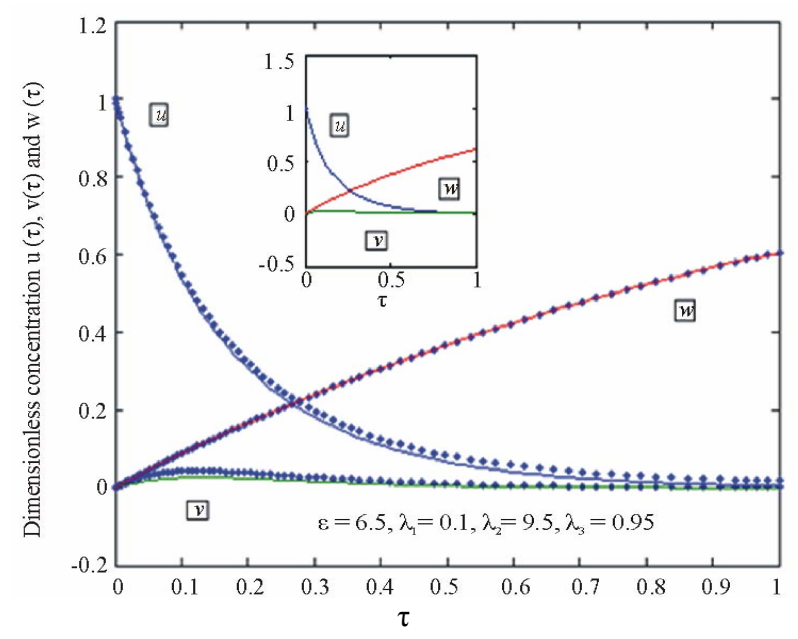

Figure 4. Time-dependent behaviour of the reactant concentration of the substrate $u(\tau)$, enzyme-substrate $v(\tau)$ and free enzyme $w(\tau)$ for $\varepsilon=6.5$,

$\lambda_{1}=0.1, \lambda_{2}=9.5$ and $\lambda_{3}=0.95$. The curves are plotted using Equations (19)-(21). The key to the graph (---) represents Equations (19)-(21) and (....) denotes simulation.

$$
\begin{array}{r}
\frac{\mathrm{d} c}{\mathrm{~d} t} \approx 0 \text { for } t>t_{c} \text { and } s \approx s_{0} \text { for } t \leq t_{c} \\
c=a b\left[\frac{l_{1}-l_{2}}{k_{1} k_{\delta} e_{0}}\right]\left[\exp \left(l_{2} t\right)-\exp \left(l_{1} t\right)\right] \\
e_{i}=e_{0}+a \exp \left(l_{1} t\right)-b \exp \left(l_{2} t\right)
\end{array}
$$

where

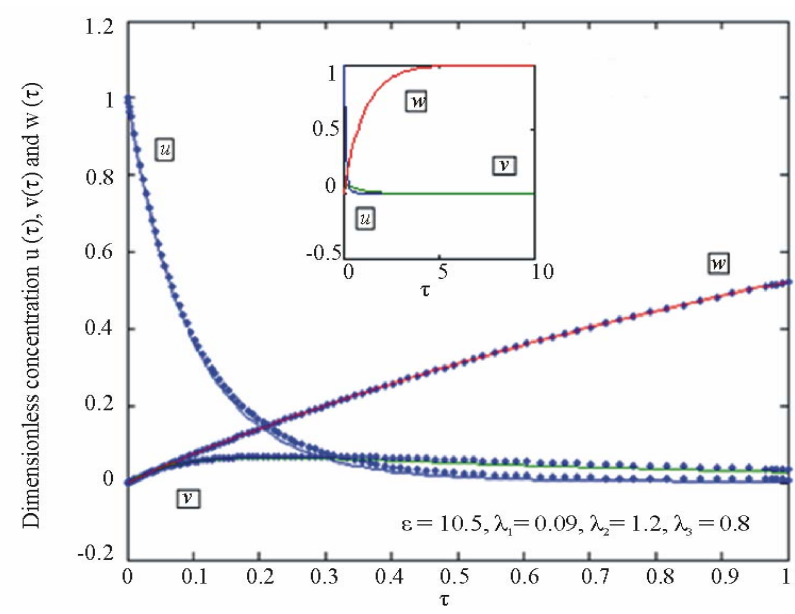

Figure 5. Time-dependent behaviour of the reactant concentration of the substrate $u(\tau)$, enzyme-substrate $v(\tau)$ and free enzyme $w(\tau)$ for $\varepsilon=10.5$,

$\lambda_{1}=0.09, \lambda_{2}=1.2$ and $\lambda_{3}=0.8$. The curves are plotted using Equations (19)-(21). The key to the graph (---) represents Equations (19)-(21) and (....) denotes simulation.

$$
\begin{gathered}
a=\frac{e_{0}\left(l_{2} / l_{1}+k_{\delta}\right)}{\left(l_{1}-l_{2}\right) / k_{1}}, \quad b=\frac{e_{0}\left(l_{1} / k_{1}+k_{\delta}\right)}{\left(l_{1}-l_{2}\right) / k}, \\
l_{1}=\frac{k_{1} k_{\delta} k_{M}}{k_{M}^{a p p}+s_{0}}+O\left(t^{2}\right), l_{2}=-k_{1}\left(k_{M}^{a p p}+s_{0}\right)+O\left(t^{2}\right)
\end{gathered}
$$

Figures 1-5 represent the dimensionless concentration of substrate $u$, enzyme-substrate complex $v$, and free enzyme $w$ for various values of parameter $\varepsilon, \lambda_{1}, \lambda_{2}$ and $\lambda_{3}$. From these figures it is inferred that the concentration of substrate $u$ follows a first-order exponential decay and it is always decreasing function from the initial value. The concentration of enzyme-substrate complex $v$ initially increases and attains its steady state value at short intervals of time for all values of rate constant. The value of free enzyme $w$ increases slowly and reaches the steady state when time is very large. The time taken the steady value depends upon the values of parameters $\varepsilon, \lambda_{1}, \lambda_{2}$ and $\lambda_{3}$. Our approximate analytical expressions of substrate, enzyme substrate and free enzyme concentration are compared with simulation results in Figures 1-5. A satisfactory agreement is noted.

\section{Conclusions}

The time dependent non-linear reaction-diffusion equation has been formulated and solved analytically and numerically. Analytical expression of substrate, enzymesubstrate and free enzyme concentrations interms of dimensionless reaction diffusion parameters $\varepsilon, \lambda_{1}, \lambda_{2}$ 
and $\lambda_{3}$ are derived using the HPM. The primary result of this work is simple approximate calculations of concentrations for all values of dimensionless parameters $\varepsilon, \lambda_{1}, \lambda_{2}$ and $\lambda_{3}$. The HPM is an extremely simple method and it is also a promising method to solve other non-linear equations. This method can be easily extended to all kinds of system of coupled non-linear equations in multi-substrate systems and networks of coupled enzyme reactions.

\section{Acknowledgements}

This work was supported by the Council of Scientific and Industrial Research (CSIR No.01 (2442)/10/EMR-II), Government of India. The authors also thank Secretary, The Madura College Board and Principal, Head of the Department of Mathematics, The Madura College, Madurai, India for their constant encouragement.

\section{References}

[1] M. P. Deutscher, "Rat Liver Glutamyl Ribonucleic Acid Synthetase. I. Purification and Evidence for Separate Enzymes for Glutamic Acid and Glutamine," Journal of Biological Chemistry, Vol. 242, No. 6, 1967, pp. 11231131.

[2] J. Hernadez-Ruiz, M. B. Amao, A. N. P Hiner, F. Garcia-Canovas and M. Acosta, "Catalase-Like Activity of Horseradish Peroxidase: Relationship to Enzyme Inactivation by $\mathrm{H}_{2} \mathrm{O}_{2}$," Biochemical Journal, Vol. 354, No. 2, 2001, pp. 107-114.

[3] J. Messens, J. C. Martins, E. Brosens, K. Van Belle, D. M. Jacobs, R. Willem and L. Wyns, "Kinetics and Active Site Dynamics of Staphylococcus Aureus Reductase," Journal of Biological Inorganic Chemistry, Vol. 7, 2002, pp. 146-156. doi:10.1007/s007750100282

[4] S. Ho and G. S. Mittal, "High Voltage Pulsed Electrical Field for Liquid Food Pasteurization," Food Reviews International, Vol. 16, No. 4, 2000, pp. 395-434. doi:10.1081/FRI-100102317

[5] C. P. Yao and R. H Levy, "Inhibition-Based Metabolic Drug-Drug Interactions: Predictions from in Vitro Data," Journal of Pharmaceutical Sciences, Vol. 91, No. 9, 2002, pp. 1923-1935. doi:10.1002/jps. 10179

[6] S. G. Waley, "Kinetics of Suicide Substrates," Biochemical Journal, Vol. 185, 1980, pp. 771-773.

[7] S. G. Waley, "Kinetics of Suicide Substrates. Practical Procedures for Determining Parameters," Biochemical Journal, Vol. 227, 1985, pp. 843-849.

[8] L. Michaelis and M. L. Menten, "Die Kinetik der Invertinwirking," Biochemische Zeitschrift, Vol. 49, 1913, pp. 333-369.

[9] G. E. Briggs and J. B. S. Haldane, "A Note on the Kinetics of Enzyme Action," Biochemical Journal, Vol. 19, No. 2, 1925, pp. 338-339.
[10] S. Schnell and S. M. Hanson, "A Test for Measuring the Effects of Enzyme Inactivation," Biophysical Chemistry, Vol. 125, No. 2-3, 2007, pp. 269-274. doi:10.1016/j.bpc.2006.08.010

[11] S. J. Li and Y. X. Liu, "An Improved Approach to Nonlinear Dynamical System Identification Using PID Neural Networks," International Journal of Nonlinear Sciences and Numerical Simulation, Vol. 7, No. 2, 2006, pp. 177-182.

[12] M. M. Mousa, S. F. Ragab and Z. Nturforsch, "Application of the Homotopy Perturbation Method to Linear and Nonlinear Schrödinger Equations," Zeitschrift für Naturforschung, Vol. 63, 2008, pp. 140-144.

[13] J. H. He, "Homotopy Perturbation Technique," Computer Methods in Applied Mechanics and Engineering, Vol. 178,1999 , pp. 257-262. doi:10.1016/S0045-7825(99)00018-3

[14] J. H. He, "Homotopy Perturbation Method: A New Nonlinear Analytical Technique," Applied Mathematics and Computation, Vol. 135, No. 1, 2003, pp. 73-79. doi:10.1016/S0096-3003(01)00312-5

[15] J. H. He, "A Simple Perturbation Approach to Blasius Equation," Applied Mathematics and Computation, Vol. 140, No. 2-3, 2003, pp. 217-222. doi:10.1016/S0096-3003(02)00189-3

[16] J. H. He, "Some Asymptotic Methods for Strongly Nonlinear Equations," International Journal of Modern Physics B, Vol. 20, No. 10, 2006, pp. 1141-1199. doi:10.1142/S0217979206033796

[17] J. H. He, G. C. Wu and F. Austin, "The Variational Iteration Method Which Should Be Followed," Nonlinear Science Letters A, Vol. 1, No. 1, 2010, pp. 1-30.

[18] J. H. He, "A Coupling Method of a Homotopy Technique and a Perturbation Technique for Non-Linear Problems," International Journal of Non-Linear Mechanics, Vol. 35, No. 1, 2000, pp. 37-43. doi:10.1016/S0020-7462(98)00085-7

[19] D. D. Ganji, M. Amini and A. Kolahdooz, "Analytical Investigation of Hyperbolic Equations via He's Methods," American Journal of Engineering and Applied Sciences, Vol. 1, No. 4, 2008, pp. 399-407.

[20] S. Loghambal and L. Rajendran, "Mathematical Modeling of Diffusion and Kinetics of Amperometric Immobilized Enzyme Electrodes," Electrochimica Acta, Vol. 55, No. 18,2010 , pp. 5230-5238. doi:10.1016/j.electacta.2010.04.050

[21] A. Meena and L. Rajendran, "Mathematical Modeling of Amperometric and Potentiometric Biosensors and System of Non-Linear Equations-Homotopy Perturbation Approach," Journal of Electroanalytical Chemistry, Vol. 644 , No. 1,2010 , pp. 50-59. doi:10.1016/j.jelechem.2010.03.027

[22] A. Eswari and L. Rajendran, "Analytical Solution of Steady State Current an Enzyme Modified Microcylinder Electrodes," Journal of Electroanalytical Chemistry, Vol. 648, No. 1, 2010, pp. 36-46. doi:10.1016/j.jelechem.2010.07.002 


\section{APPENDIX A}

Solution of the Equations (15)-(18) using Homotopy perturbation method. In this Appendix, we indicate how Equations (19)-(21) in this paper are derived. Furthermore, a Homotopy was constructed to determine the solution of Equations (15)-(17).

$$
\begin{gathered}
(1-p)\left[\frac{\mathrm{d} u}{\mathrm{~d} \tau}+u \varepsilon\right]+p\left[\frac{\mathrm{d} u}{\mathrm{~d} \tau}+u \varepsilon-\lambda_{1} \varepsilon v-\varepsilon u v-\varepsilon u w\right]=0 \\
(1-p)\left[\frac{\mathrm{d} v}{\mathrm{~d} \tau}+\lambda_{2} v\right]+p\left[\frac{\mathrm{d} v}{\mathrm{~d} \tau}+\lambda_{2} v-u+u v+u w\right]=0 \\
(1-p)\left[\frac{\mathrm{d} w}{\mathrm{~d} \tau}+\lambda_{3} w\right]+p\left[\frac{\mathrm{d} w}{\mathrm{~d} \tau}+\lambda_{3} w-\lambda_{3}+\lambda_{3} v\right]=0
\end{gathered}
$$

The initial approximations are as follows:

$$
u(0)=1, v(0)=0 \text { and } w(0)=0
$$

Approximate solutions of (A1), (A2) and (A3) are

$$
\begin{aligned}
& u=u_{0}+p u_{1}+p^{2} u_{2}+p^{3} u_{3}+ \\
& v=v_{0}+p v_{1}+p^{2} v_{2}+p^{3} v_{3}+
\end{aligned}
$$

and

$$
w=w_{0}+p w_{1}+p^{2} w_{2}+p^{3} w_{3}+.
$$

Substituting Equations (A5)-(A7) in Equations (A1)-(A3) and comparing the coefficients of like powers of $p$, we can obtain the following differential equations.

$$
\begin{gathered}
p^{0}: \frac{\mathrm{d} u_{0}}{\mathrm{~d} \tau}+\varepsilon u_{0}=0 \\
p^{1}: \frac{d u_{1}}{d \tau}+\varepsilon u_{1}-\lambda_{1} \varepsilon v_{0}-\varepsilon u_{0} v_{0}-\varepsilon u_{0} w_{0}=0 \\
p^{2}: \frac{\mathrm{d} u_{2}}{\mathrm{~d} \tau}+\varepsilon u_{2}+\varepsilon u_{1}-\lambda_{1} \varepsilon v_{1}-\varepsilon u_{0} w_{1}-\varepsilon u_{1} w_{0}=0
\end{gathered}
$$

and

$$
\begin{gathered}
p^{0}: \frac{\mathrm{d} v_{0}}{\mathrm{~d} \tau}+\lambda_{2} v_{0}=0 \\
p^{1}: \frac{\mathrm{d} v_{1}}{\mathrm{~d} \tau}+\lambda_{2} v_{1}-u_{0}+u_{0} v_{0}+u_{0} w_{0}=0 \\
p^{2}: \frac{\mathrm{d} v_{2}}{\mathrm{~d} \tau}+\lambda_{2} v_{2}-u_{1}+u_{0} v_{1}+u_{1} v_{0}+u_{0} w_{1}+u_{1} w_{0}=0
\end{gathered}
$$

and

$$
\begin{aligned}
& p^{0}: \frac{\mathrm{d} w_{0}}{\mathrm{~d} \tau}+\lambda_{3} w_{0}=0 \\
& p^{1}: \frac{\mathrm{d} w_{1}}{\mathrm{~d} \tau}+\lambda_{3} w_{1}-\lambda_{3}+\lambda_{3} v_{0}=0 \\
& p^{2}: \frac{\mathrm{d} w_{2}}{\mathrm{~d} \tau}+\lambda_{3} w_{2}+\lambda_{3} v_{1}=0
\end{aligned}
$$

Solving the Equations (A8)-(A16), and using the boundary conditions (A4), we can find the following results. 


$$
\begin{gathered}
u_{0}(\tau)=e^{-\varepsilon \tau} \\
u_{1}(\tau)=0 \\
u_{2}(\tau)=\frac{\lambda_{1} \varepsilon \tau e^{-\varepsilon \tau}}{\lambda_{2}-\varepsilon}+\frac{\lambda_{1} \varepsilon\left[e^{-\lambda_{2} \tau}-e^{-\varepsilon \tau}\right]}{\left(\lambda_{2}-\varepsilon\right)^{2}}+\varepsilon \tau e^{-\varepsilon \tau}+\frac{\varepsilon\left[e^{-\left(\lambda_{3}+\varepsilon\right) \tau}-e^{-\varepsilon \tau}\right]}{\lambda_{3}}
\end{gathered}
$$

and

$$
\begin{gathered}
v_{0}(\tau)=0 \\
v_{1}(\tau)=\frac{e^{-\varepsilon \tau}-e^{-\lambda_{2} \tau}}{\lambda_{2}-\varepsilon} \\
\nu_{2}(\tau)=\frac{1}{\lambda_{2}-\varepsilon}\left[\frac{e^{-\lambda_{2} \tau}}{\varepsilon}-\frac{e^{-\left(\lambda_{1}+\varepsilon\right) \tau}}{\varepsilon}+\frac{e^{-\lambda_{2} \tau}}{\lambda_{2}-2 \varepsilon}-\frac{e^{-2 \varepsilon \tau}}{\lambda_{2}-2 \varepsilon}\right]+\left[\frac{e^{-\left(\lambda_{3}+\varepsilon\right) \tau}-e^{-\lambda_{2} \tau}}{\lambda_{2}-\lambda_{3}-\varepsilon}\right]+\left[\frac{e^{-\lambda_{2} \tau}-e^{-\varepsilon \tau}}{\lambda_{2}-\varepsilon}\right]
\end{gathered}
$$

and

$$
\begin{gathered}
w_{0}(\tau)=0 \\
w_{1}(\tau)=1-e^{-\lambda_{3} \tau} \\
w_{2}(\tau)=\frac{\lambda_{3} e^{-\lambda_{3} \tau}}{\left(\lambda_{2}-\varepsilon\right)\left(\lambda_{3}-\varepsilon\right)}-\frac{\lambda_{3} e^{-\varepsilon \tau}}{\left(\lambda_{2}-\varepsilon\right)\left(\lambda_{3}-\varepsilon\right)}+\frac{\lambda_{3} e^{-\lambda_{2} \tau}}{\left(\lambda_{2}-\varepsilon\right)\left(\lambda_{3}-\lambda_{2}\right)}-\frac{e^{-\lambda_{3} \tau}}{\left(\lambda_{2}-\varepsilon\right)\left(\lambda_{3}-\lambda_{2}\right)}
\end{gathered}
$$

According to the HPM, we can conclude that

$$
\begin{gathered}
u(\tau)=\lim _{p \rightarrow 1} u(\tau)=u_{0}+u_{1}+u_{2}+\ldots \ldots \ldots . \\
v(\tau)=\lim _{p \rightarrow 1} v(\tau)=v_{0}+v_{1}+v_{2}+\ldots \ldots \ldots \\
w(\tau)=\lim _{p \rightarrow 1} w(\tau)=w_{0}+w_{1}+w_{2}+\ldots \ldots \ldots .
\end{gathered}
$$

Using Equations (A17)-(A19) in Equation (A26), Equations (A20)-(A22) in Equations (A27) and (A23)-(A25) in Equation (A28), we obtain the final results as described in Equations (19)-(21).

\section{APPENDIX B}

Scilab program to find the solutions of the Equations (15)-(18)

function main123456

options= odeset ('RelTol', 1e-6, 'Stats', 'on' $)$;

\%initial conditions

$\mathrm{x} \Theta=[1 ; 0 ; 0]$;

$\operatorname{tspan}=\left[\begin{array}{ll}0 & 1\end{array}\right] ;$

tic

$[\mathrm{t}, \mathrm{x}]=\operatorname{ode} 45(@$ TestFunction, tspan, $\mathrm{x} \odot$, options);

toc

figure

hold on

$\operatorname{plot}(t, x(:, 1))$

$p \operatorname{plot}\left(t, x(:, 2),{ }^{\prime} \cdot{ }^{\prime}\right)$

$\operatorname{plot}(t, x(:, 3))$

legend ('x1', ' $\times 2$ ')

ylabel( ' $x$ ' )

xlabel(' $t$ ')

return

function $\left[d x \_d t\right]=$ TestFunction $(t, x)$ 
$a=6.5 ; b=0.4 ; c=4 ; d=0.3 ;$

$\mathrm{dx}$ _dt $(1)=-\mathrm{a}^{*} \mathrm{x}(1)+\mathrm{a}{ }^{*} \mathrm{x}(1){ }^{*} \mathrm{x}(2)+\mathrm{a}{ }^{*} \mathrm{x}(1){ }^{*} \mathrm{x}(3)+\mathrm{a}{ }^{*} \mathrm{~b} \times(2)$;

$\mathrm{dx}$ _dt (2) $=x(1)-x(1) * x(2)-x(1){ }^{*} x(3)-c^{*} x(2)$;

$d x \_d t(3)=d-d^{*} x(2)-d^{*} x(3)$;

$\mathrm{dx} \_\mathrm{dt}=\mathrm{dx} \_\mathrm{dt} \mathrm{t}^{\prime}$;

return

\section{APPENDIX C}

$\begin{array}{ll}\begin{array}{ll}\text { Nomenclature } \\ u\end{array} & \text { Dimensionless Substrate concentation (None) } \\ v & \text { Dimensionless Enzyme-substrate concentration (None) } \\ w & \text { Dimensionless free enzyme concentration (None) } \\ K_{M} & \text { Michaelis-Menten constant }(\mu \mathrm{M}) \\ K_{S} & \text { Equlibrium dissociation constant }(\mu \mathrm{M}) \\ K_{\delta} & \text { Enzyme inactivation constant }(\mu \mathrm{M}) \\ k_{c a t} & \text { Unimolecular rate constant }(\mu \mathrm{M}) \\ \lambda_{1} & \\ \lambda_{2} & \text { Dimensionless Equlibrium dissociation constant (None) } \\ \lambda_{3} & \text { Dimensionless Michaelis-Menten constant (None) } \\ \varepsilon & \text { Dimensionless enzyme inactivation constant (None) } \\ \tau & \text { Dimensionless reaction diffusion constant (None) }\end{array}$

